Journal of Mathematics and Statistics 6 (3): 350-356, 2010

ISSN 1549-3644

(C) 2010 Science Publications

\title{
An Application of Multivariate Analysis in Modeling Students Placement in Nigerian Higher Institutions
}

\author{
${ }^{1}$ Ali Idrisa Gambo and ${ }^{2}$ Mohammed Waziri Yusuf \\ ${ }^{1}$ Department of Statistics, Central Bank of Nigeria, Abuja \\ ${ }^{2}$ Department of Mathematics, Faculty of Science, \\ University Putra Malaysia 43400 Serdang, Malaysia
}

\begin{abstract}
Problem statement: Students' placement into courses of study in most (if not all) tertiary institutions in Nigeria had long been a problematic exercise. Most students get placed into courses of study they are ill suited to and then for which they are ill prepared. Approach: This research focused on the polytechnic, taking the newest federal polytechnic as a case study, that is, Federal Polytechnic Damaturu. The research tries to design a predictive model for the purpose of placing incoming students from the Pre-National Diploma (Pre-ND) programme, into courses of study based on the students' performance at the Pre-ND level. The classification model sought to assign a student into a course of study, through the analysis of subjects scores at the Pre-ND level. Results: Discriminate analysis technique was applied and results obtained indicated different subjects in the different courses as the strongest contributors for the placement of students into different courses of study. The science courses have similar subjects as strong contributors, while the management courses share the same subjects mix as contributors to a student's placement into a course. Conclusion: The natures of the models constitute discriminate factors and the potential for its improvement in future are also discussed in the research.
\end{abstract}

Key words: Multivariate analysis, modeling and discriminant analysis

\section{INTRODUCTION}

The Nigerian Tertiary Education system is composed of two tiers; the universities and the polytechnics (including Colleges of Education). The Polytechnic sector emphasizes Technical and Vocational Education for middle level cadre in Technical and vocational sectors of the Nigerian Industrial and Business Sectors. Over the years, (since 1976), the Polytechnic s have proven to be a viable alternative to the University sector. Infect it offers the entire degree and sub degree course for the prospective applicants. But recently, there is high redundancy rate and student retention in the Polytechnics. This coincided with the downward spiral in the quality and quantity of the graduates of the Nigerian tertiary institutions. Worst hit are states with precarious position on the Educational development index. At the bottom of the Educational development index are New States (created in 1991). The polytechnics offer a graduated entry point for a lot of applicants, by going through a pre-degree programme much a la A-levels, for one year. The main programmes offered, confers a National Diploma (ND) on the graduate after two years of study and Higher National Diploma (HND) after another two years of study. Between the National Diploma and the Higher National Diploma (HND) there is one year interregnum for practical Industrial attachment.

All polytechnics $\mathrm{n}$ the country are accredited and supervised by the National Board for Technical Education (NBTE) for programme quality and standards.

The Federal Polytechnic Damaturu is one the newest Federal Polytechnics in Nigeria. It was established in 1992. The Institution is fully accredited by the National Board for Technical Education (NBTE) to run and award National Diplomas and awards of Higher National Diploma programmes in the following disciplines:

- Statistics (HND Statistics)

- Secretariat Studies (HND Sec. Studies)

- Accounting (HND Accounting)

- Business Management (HND Business)

- Science Lab Technology (HND SLT Chemistry option and Bio-Chemistry option)

The polytechnic maintains two broad admission schedules, namely:

Corresponding Author: Ali Idrisa Gambo, Department of Statistics, Central Bank of Nigeria, Abuja 
- Through the Joint Admission and Matriculation Board Examination, known as Poly JAMB

- Through the Pre-National Diploma programmer known as the Pre-ND

Students can be admitted through either of these schedules. Those admitted through Poly JAMB, get placed directly to their respective courses in their year one, otherwise known as National Diploma one or ND1, provided they meet the necessary entry requirements in terms of WAEC qualifications. While those that came through the Pre-ND get placed into their respective courses after a successful completion of their one year Pre-ND programme.

The Pre-ND programmer is an internally run programmer by the Polytechnic, that is aimed at remedying some of the deficiencies in the entry requirements of the students. As such it serves as the main entry point into the diploma programmers, after a successful completion of the Pre-ND programmer.

The Pre-ND programmer is broadly divided into two categories:

- Pre- ND Science

- Pre-ND Management

The Pre-ND science caters for students admitted into the following disciplines:

- Statistics

- Computer Sciences

- Science Lab Technology (SLT)

- Mechanical Engineering

- Electrical Engineering

- Civil Engineering

- Surveying

On the other hand, the Pre-ND management caters for students admitted into the following disciplines:

- Business Studies

- Banking and Finance

- Secretariat Studies

- Accounting

- Estate Management

A student is adjudged to have passed the Pre-ND programme by the academic Board, if he/she passed Five Subjects, including Mathematics, English Language and three other subjects which are regarded as core subjects depending on his/her categorization, that is whether science or management, with an average score of 50 marks graded as ' $C$ '. The average score of both the first and second semester scores are taken and presented at the Academic Board.

Over the years it was observed that the placed ND students were having mass failures in their ND programmers. This generally watered down the programmer by increasing the number of repeating students and carry over students. Therefore a means of placing students into the right programmer is needed.

The principal objective of this study is to develop a statistical model that can be used to set a standard for placing Pre-ND students into the right ND programmer based on their Pre-ND results.

The significance of this study, among others, is that the designed rule or Model constructed will provide:

- A standardized rule, which is objective, for the proper placement of students into diploma programmers

- A handy tool for admission and placement problems

- A means of reducing the teaching overload of lecturers through the reduction of incidence of carry over and repeat, using proper placement procedure

Multivariate analysis provides the statistical techniques that can be used by the researcher to analyse complex sets of data. It can handle the analysis of problems that involve independent variables and several dependent variables, which may be correlated to each other to varying degrees. The ready availability of software application programmes, which can handle the complexity of large multivariate data sets, has increased and popularized the use of multivariate analysis. For an analysis involving multivariate methods, an appropriate data set is composed of values related to a number of variables for a number of subjects. Accordingly appropriate data sets may be organized as; data matrix, a correlation matrix, a variance-covariance matrix, a sum of squares and cross products matrix or sequence residuals (Tabachnick and Fidell, 2000).

\section{MATERIALS AND METHODS}

Discriminant Analysis (DA): As a multivariate technique is concerned with the investigation of the relationships between a known grouping of the data and the variables recorded i.e., for investigating or grouping cases with similar characteristics and to identify a mathematical function which in turn exhibits differences among observed groups. The outcome of the analysis is an allocation rule, often but not 


\section{J. Math. \& Stat., 6 (3): 350-356, 2010}

necessarily, associated with the values taken by a discriminate function. This rule can then be used for assigning to their appropriate groups the members of subsequent samples. To achieve this, a linear discriminate function is developed via maximizing the ratio of between-group variation to within-group variation (Kinnear and Gray, 1999).

Ideas associated with Discriminant analysis can be traced back to the 1920's, through the works completed by English statistician Karl Pearson (1857-1936), who translated multivariate inter group distance into linear combination of variables to aid in inter group discrimination (Anderson, 1996).

Since then, Discriminant analysis has been applied to a wide variety of disciplines. For example, earlier applications, financial ratio analysis to make predictions of company bankruptcy (Altman, 1968). The technique was subsequently used to predict company financial performance by many researchers (Deakin, 1972; Johnson, 1970). Lennox (1999) also used the technique to determine the factors responsible for a company's bankruptcy. Wong and Holt (2003) also applied Discriminant analysis to develop a contractor classification model that linked clients' evaluation aspirations with contractor potential performance, in the construction industry.

Sharma and Palawal (2006) used a rotational Linear discriminant analysis to identify a linear transformation orientation that best project the feature vectors to a subspace in such a way that the overlapping between the different subspaces classes is minimum.

Discriminant analysis is concerned with the problem of assigning an unknown observation to a group with low error rate, therefore the basic idea of Discriminant analysis is the designing of a rule or function which will determine whether groups differ with regards to the mean of a variable and to use that variable to predict group membership of new cases. The design of such a rule is called Discriminant Function Analysis.

Chan et al. (2010) applied two multivariate tools on comparative basis in facial biometrics feature extraction in automatic recognition. Principal Component Analysis PCA) and Linear Discriminant Analysis. The two methods were applied to a large database of images, consisting of facial features and other biometric; the Linear Discriminant Analysis outperformed the PCA in biometrics feature extraction.

Chentir et al. (2008) showed how to apply discriminant analysis to identify stress syllables of accented words on the basis of fundamental frequency in Standard Arabic words. The approach utilizes the acoustic parameter of energy used by means of linear discrimination.
Martinez (2001) also reported on the ability of Discriminant analysis as a statistical predictive tool in placing student into the right course that matches students' capabilities after conducting a series of standardized tests on the students after admission. Subsequent researches focused on not only on predicting student's placement through the use of performance, but also on other factors that might influence a student's performance in the school. Dyer et al. (2002) and in identifying students at risk, that is identifying a student at risk of failure of the academic programme, because of the student's social background, status, religious inclination, race and other variables worth considering because of it's political and social implications in a multi cultural and heterogeneous society (Thomas et al., 1996). Pyryt (1986) used Discriminant analysis to illustrate how the technique can maximize the effectiveness and efficiency of screening procedures for identifying intellectually gifted children.

The main crux of multivariate analysis is to explain variation according to one or more of the variables respectively. The aim of most applied studies is concerned with variation on some characteristics. This variation can be explained according to one or more of the following:

- Determination of the nature and degree of association between a set of criterion variables and a set of predictor variables, often called "dependent" and "independent" variables respectively

- Finding a function or formula by which one can estimate values of the criterion variables

- Placing confidence limits or intervals on the estimates via the test of statistical significance

- Among the several techniques available in the application of multivariate analysis, one would be singled out for application in this particular study, namely; Discriminant Analysis (DA)

Discriminant Function Analysis: Discriminant function Analysis is used to classify cases into the values of a categorical dependent variable, usually a dichotomy. If Discriminant function analysis is effective for a set data, the classification table of correct and incorrect estimates will yield a high percentage of correct classification. (Tabachnick and Fidell, 2000)

In order to obtain an optimal Discriminant function i.e., to provide a good classification model, the following assumptions about the data must be met:

- The data must have a multivariate normal distribution

- There must be equal group population covariance matrices in the data i.e., homogeneity of variances/covariances 
- Multicollinearity among the variables must be avoided

Discriminant analysis as an analytical tool has some desirable advantages of being able to achieve the following:

- Classification of cases into groups using a Discriminant prediction equation

- To determine the percentage of variance in the dependent variable explained by the independents over and above the variance accounted for by control variables, using sequential Discriminant analysis

- To assess the relative importance of the independent variables in classifying the dependent variable

- Consideration of Multicollinearity (close interrelationships) between independent variables.

- Can accommodate the entire profile (level of measurement) of different types of variables (i.e., ratio, interval and nominal data)

- To determine the most parsimonious way (the fewest dimensions) to distinguish between groups.

- To infer the meaning of the dimensions, which distinguish groups, based on Discriminant loadings

- It is straightforward function, in the sense that the derived final Discriminant factors profile is statistically significant for determining the relative contribution of each variable to the discriminating power

\section{Discriminant analysis has two validation steps:}

- An F-test (Wilks lambda), is used to test if the Discriminant model as a whole is significant

- If the F-test shows significance, then the individual independent variables are assessed to see which differ significantly in mean by group and these are used to classify the dependent variable.

Theory: Discriminant analysis finds a set of linear combinations of the variables, whose values are as close as possible, within groups and as far apart as possible, between groups. The linear combinations are called Discriminant Functions. Thus, a Discriminant function is a linear combination of the Discriminant variables. It has the following mathematical form:

$$
\mathrm{Y}_{\mathrm{km}}=\mathrm{b}_{\mathrm{o}}+\mathrm{b}_{1} \mathrm{X}_{1 \mathrm{~km}}+\mathrm{b}_{2} \mathrm{X}_{2 \mathrm{~km}}+\ldots \ldots \ldots+\mathrm{b}_{\mathrm{p}} \mathrm{X}_{\mathrm{pkm}}
$$

Where:

$\mathrm{Y}_{\mathrm{km}}=$ The value (score) on the canonical Discriminant function for case $m$ in the group
$X_{\mathrm{ikm}}=$ The value on the Discriminant variable $X_{\mathrm{i}}$ for case $\mathrm{m}$ in group $\mathrm{k}$

$b_{i}=$ Coefficients which produce the desired characteristics in the function

The coefficients for the 1st Discriminant function are derived so as to maximize the differences between the group means. The coefficients for the 2 nd Discriminant function are derived to maximize the difference between means, subject to the constraint that the values on the 2nd Discriminant function are not correlated with the values on the 1 st Discriminant function and so on. In other words, the 2nd Discriminant is orthogonal to the 1 st and the $3 \mathrm{rd}$ Discriminant function is orthogonal to the 2nd and so on. The maximum number of unique functions that can be derived is equal to the number of the groups minus one or equal to the number of discriminating variables, whichever is less. The Discriminant functions are generated from a sample of individuals (or cases) for which group memberships is known. The functions can then be applied to new cases with measurements on the same set of variables, but unknown group memberships.

\section{RESULTS}

The data for this study is the Pre-ND student final score of the Federal Polytechnic Damaturu, obtained from the average of the scores for the 1st and 2nd semesters. All the subjects registered for by a student in every course specialization or Department would be used in constructing the Discriminant function.

The data was analyzed using a computer statistical package, SPSS. It was used because of the various support features of the package for Discriminant analysis.

A Matrix of the student's scores is constructed for the data analysis.

\section{Variables:}

English, Math, Physics, Chemistry, Tec Draw, Bricklaying.

Objects:

1

2

3

$\mathrm{N}$ 
Computationally, the linear Discriminant function takes the form:

$$
\mathrm{D}=\mathrm{a}_{1} \mathrm{x}_{1}+\mathrm{a}_{2} \mathrm{x}_{2}+\ldots \ldots \ldots \ldots+\mathrm{a}_{\mathrm{p}} \mathrm{x}_{\mathrm{p}}
$$

There are $\mathrm{Q}$ (equal G-1 or $\mathrm{P}$ ) possible Discriminant functions corresponding to the dimensions of the data set. Since the data matrix is an $\mathrm{N} \times \mathrm{P}$ data matrix with $\mathrm{G}$ groups, where $\mathrm{N}$ is the number of sampling entities (rows) and $\mathrm{P}$ is the number of variables (columns), $\mathrm{X}_{1}, \mathrm{X}_{2}, \ldots \ldots \ldots . . \mathrm{X}_{\mathrm{P}}$ for each sample member. There are $\mathrm{m}$ random samples from different groups with sizes $a_{1}$, $\mathrm{a}_{2}, \ldots \ldots \ldots . . \mathrm{a}_{\mathrm{m}}$.

Therefore the linear Discriminant function is:

$D_{1}=a_{11} X+a_{12} X_{2}+\ldots \ldots \ldots \ldots \ldots \ldots \ldots+a_{1 p} X_{p}$

$\mathrm{D}_{2}=\mathrm{a}_{21} \mathrm{X}+\mathrm{a}_{22} \mathrm{X}_{2}+\ldots \ldots \ldots \ldots \ldots \ldots \ldots \ldots+\mathrm{a}_{2 \mathrm{p}} \mathrm{X}_{\mathrm{p}}$

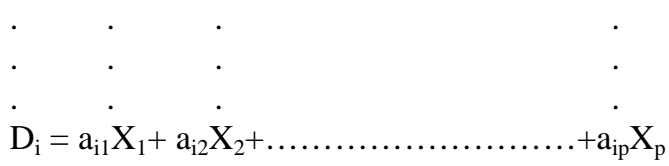

Changing the coefficients a to $\beta$ the linear Discriminant function becomes:

$\mathrm{D}_{1}=\beta_{\mathrm{i} 1} \mathrm{X}_{1}+\beta_{\mathrm{i} 2} \mathrm{X}_{2}+\ldots \ldots \ldots \ldots \ldots \ldots \ldots \ldots \ldots+\beta_{\mathrm{ip}} \mathrm{X}_{\mathrm{p}}$

Where:

$D_{i}=$ The score of the Discriminant function of the ith respondent

$\mathrm{X}_{\mathrm{p}}=$ The values of the $\mathrm{P}$ discriminating variables

$\mathrm{B}_{\mathrm{ip}}=$ The standardized weights or coefficients to be estimated for each discriminating variable

The standardized weighting coefficients $\left(B_{i p}\right)$ reflect the relative importance of each discriminating variable $\left(\mathrm{X}_{\mathrm{p}}\right)$. Variables with relatively larger $\mathrm{B}_{\mathrm{ip}}$ contribute more to the discrimination of groups. The Wilk's lambda as a statistic is used to gauge the importance of the discriminant function, the smaller the value, the better the discriminating power of the function. The Eigenvalues and canonical correlation are two other indices to indicate the choice of a discriminant function, between the functions derived. A large Eigenvalue is associated with a strong function and it indicates the proportion of variance explained (between groups sums of squares divided by within groups' sum of squares). The canonical correlation is the correlation between the discriminant scores and the levels of the dependent variable while a high correlation indicate a function that discriminates well.

Once the discriminant function had been computed, a discriminant scores can be constructed for all the course specializations, using the means of the independent variables in the function. The values obtained at the end of the derivation of the function gives the point for classifying a student if he /she meet the cutoff point.

The presence of negative coefficients in the Discriminant Function tends to lower the cutoff points and contribute less to the function. In almost all the courses English language was depicted as having a negative coefficient, thereby contributing marginally to the overall discriminant function. Secondly, the Independent variables that recorded negative discriminant coefficient in my opinion indicate the level of fear, apprehension and the general misconception of the concept being taught in that particular subject, by the students for that particular subject, inasmuch as the students wish to pass that particular course. This normally leads to poor performance in that particular subject. Also the magnitude of the coefficient of the independent variables also shows the relative size of the students' mental and academic conceptualization of the concept of a particular subject.

\section{DISCUSSION}

Discriminant scores derived from the functions can be used to predict/determine the groupings of unclassified cases. Secondly, it can also serve the purpose of determining an individual's status, vis-a-vis his/her academic qualifications and the choice area's entry requirements (Table 1) Going through the Assignment rule.

Table 1: Assignment rules

\begin{tabular}{lccc}
\hline Courses & Assign candidate to course if Di $>\lambda$ & Reject candidate if $\mathrm{Di}<\boldsymbol{\lambda}$ & Assign candidate conditionally if $\mathrm{Di}=\boldsymbol{\lambda}$ \\
\hline Computer Science & 53.47 & 53.47 & 53.47 \\
Statistics & -1.81 & -1.81 & -1.81 \\
SLT & 60.85 & 60.85 & 60.85 \\
Survey and Geo Informatics & 299.33 & 299.33 & 299.33 \\
Civil engineering & 71.77 & 71.77 & 71.77 \\
Mechanical Engineering & 153.94 & 153.94 & 153.94 \\
Electrical Engineering & 25.27 & 25.27 & 25.27 \\
Accounting & 78.52 & 78.52 & 78.52 \\
Banking and Finance & 84.36 & 84.36 & 84.36 \\
Business Studies & 55.97 & 55.97 & 55.97 \\
Secretariat Studies & 74.37 & 74.37 & 74.37 \\
Estate Management & 96.06 & 96.06 & 96.06 \\
\hline
\end{tabular}


In science based course specializations, the courses of Computer Science, Statistics and Science Lab Tech (SLT) all reported a negative coefficient for the independent variable English. This may be due to the weak academic background of the students in English. Also of particular note, in the statistics course option is a situation where a negative value was obtained for the Discriminant Function. For this case, the Wilks lambda with a value of 0.890 was considered too high. The wilks lambda should be close to zero for a good discriminant function. In fact all the indices of measuring a function (including, large eigenvalue and high canonical correlation) showed the obtained function as inefficient on closer examination of the original data, it was discovered that, there was ambivalence among the students, some students registered for physics, rather than geography, as was the case with the majority, while others registered for both.

The presence of negative coefficients in the Discriminant Function tends to lower the cutoff points and contribute less to the function. In almost all the courses English language was depicted as having a negative coefficient, thereby contributing marginally to the overall discriminant function. Secondly, the Independent variables that recorded negative discriminant coefficient in my opinion indicate the level of fear, apprehension and the general misconception of the concept being taught in that particular subject, by the students for that particular subject, inasmuch as the students wish to pass that particular course. This normally leads to poor performance in that particular subject.

Also the magnitude of the coefficient of the independent variables also shows the relative size of the students' mental and academic conceptualization of the concept of a particular subject.

\section{CONCLUSION}

Discriminant scores derived from the functions can be used to predict/determine the groupings of unclassified cases. Secondly, it can also serve the purpose of determining an individual's status, vis-a vis his/her academic qualifications and the choice area's entry requirements.

The main objective of this research exercise is to design a particular allocation rule or prediction criteria that will allow the school involved to choose the best possible course of study for a student admitted through the pre-ND programme. This allocation rule was designed using multivariate technique namely: Discriminant Analysis.

English language being the language of instruction is giving the students a tough time towards understanding it and hence further compounding their understanding of other subjects. Core subjects with negative coefficient depict the weakness of student's academic background with respect to such subjects. Therefore a special intervention programme should be formulated and be put in place, to rescue such students as they are clearly at risk with respect to such subjects. This will go a long way in helping the polytechnic in achieving its primary function of churning out technically sound graduates.

\section{REFERENCES}

Altman, E.I., 1968. Financial ratios, discriminant analysis and the predicting of corporate bankruptcy. J. Finance, 23: 589-609. http://www.bus.tu.ac.th/department/thai/download/ news/957/Altman_1968.pdf

Anderson, T.W., 1996. R.A. Fisher and multivariate analysis. Stat. Sci., 11: 20-34. http://www.jstor.org/stable/2246198

Chentir, A., M. Guerti and D.J. Hirst, 2008. Classification by discriminant analysis of energy in view of the detection of accented syllables in standard Arabic. J. Comput. Sci., 4: 668-673. http://www.scipub.org/fulltext/jcs/jcs48668-673.pdf

Chan, L.H., S.H. Salleh and C.M. Ting, 2010. Face biometrics based on principal component analysis and linear discriminant analysis. J. Comput. Sci., 6: 693-699. http://www.scipub.org/fulltext/jcs/jcs67691-698.pdf

Deakin, E.B., 1972. A discriminant analysis of predictors of business failure. J. Account. Res., 10: 167-179. http://www.jstor.org/stable/2490225

Dyer, J.E., L.M. Breja and P.S.H. Wittler, 2002. Predictors of student retention in colleges of agriculture. ERIC.

Johnson, C.G., 1970. Ratio analysis and the prediction of firm failure. J. Finance, 25: 1166-1168. http://www.jstor.org/stable/2325590

Kinnear, P.R. and C.D. Gray, 1999. SPSS for Windows Made Simple. 3rd Edn., Psychology Press, Hove, ISBN: 0863776116, pp: 386.

Lennox, C., 1999. Failing companies: A Re-evaluation of logit, probit and discriminant analysis approaches. J. Econ. Bus., 51: 347-364. DOI: 10.1016/S0148-6195(99)00009-0.

Martinez, D., 2001. Predicting student outcomes using discriminant function analysis. Proceeding of the 39th Annual Meeting of the Research and Planning Group, May 2-4, ERIC, Lake Arrowhead, CA., pp: 1-22. 
Pyryt, M.C., 1986. Using discriminant analysis to identify gifted children. J. Educ. Gifted, 9: 233-238.

Sharma, A. and K.K. Paliwal, 2006. Rotational linear discriminant analysis using Bayes rule for dimensionality reduction. J. Comput. Sci., 2: 754-757. http://www.scipub.org/fulltext/jcs/jcs29754-757.pdf

Tabachnick, B.G. and L.S. Fidell, 2000. Using Multivariate Statistics. 4th Edn., Allyn and Bacon, Boston, ISBN: 10: 0321056779, pp: 966.

Thomas, E.W. M.J. Marr, A. Thomas, R.M. Hume and N. Walker, 1996. Using discriminant analysis to identify students at risk. Proceedings of the 26th Annual Frontiers in Education Conference, Nov. 69, IEEE Xplore Press, USA., pp: 185-188. DOI: 10.1109/FIE.1996.569940
Wong, C. and G. Holt, 2003. Developing a contractor classification model using a multivariate discriminant approach. RICS Foundation. http://www.rics.org/site/scripts/download_info.asp $\mathrm{x}$ ?fileID $=2926 \&$ categoryID $=450$ 